Commun. Korean Math. Soc. 26 (2011), No. 4, pp. 543-549

http://dx.doi.org/10.4134/CKMS.2011.26.4.543

\title{
FREE ALGEBRAS OVER A POSET IN VARIETIES
}

\author{
Aldo Figallo, Jr. And Alicia Ziliani
}

\begin{abstract}
In 1945, the notion of free lattice over a poset was introduced by R. Dilworth (Trans. Am. Math. Soc. 57 (1945), 123-154). In this note, a construction of the free algebra over a poset in varieties finitely generated is shown. Finally, this result is applied to different classes of algebras.
\end{abstract}

\section{Introduction}

In 1945, R. Dilworth ([7]) introduced the notion of free lattice over a poset. Later on, this subject was studied by many authors and in different classes of algebras. Analyzing some of them, we realized that there are certain similarities, not only in the construction but also in the structure of the varieties considered. In this sense we can assert that:

(i) Each variety is generated by an algebra and it has an underlying ordered structure definable by means of certain equations in terms of the operations of this algebra.

(ii) If $I$ is an ordered set and $A$ is the algebra which generates the variety, then the first step in the construction of the free algebra $L(I)$ over $I$ is to consider the set of all increasing functions from $I$ to $A$.

(iii) All the constructions mentioned above are strongly based on the fact that the algebras of these varieties can be represented by algebras of sets.

In what follows, we will focus on varieties of algebras that have an underlying ordered structure definable by means of certain equations $p_{i}(x, y)=q_{i}(x, y)$, $1 \leq i \leq n$ which are in terms of the operations of the algebra. For example, that is the case of Hilbert algebras ([6]) where the order is given by a single equation where $p_{1}(x, y)=x \rightarrow y$ and $q_{1}(x, y)=x \rightarrow x$ or that of distributive lattices in which we can consider $p_{1}(x, y)=x \wedge y, q_{1}(x, y)=x$ (or equivalently by $\left.p_{1}(x, y)=x \vee y, q_{1}(x, y)=y\right)$.

Received May 26, 2010; Revised March 31, 2011.

2010 Mathematics Subject Classification. 08B20, 06A12, 03G25.

Key words and phrases. free algebras, free algebras over a poset, varieties finitely generated. 
Let $\mathcal{V}$ be a variety of algebras under the above conditions. Then, the notion of free algebra over a poset $I$ in $\mathcal{V}$ can be defined as follows.

Definition 1. A $\mathcal{V}$-algebra $L_{\mathcal{V}}(I)$ is free over $I$ if it verifies these conditions:

(L1) there is an order-embedding $g: I \rightarrow L_{\mathcal{V}}(I)$ such that $L_{\mathcal{V}}(I)=[g(I)] \mathcal{v}$, where $[X] \mathcal{V}$ is the $\mathcal{V}$-subalgebra of $L_{\mathcal{V}}(I)$ generated by $X$,

(L2) for each $\mathcal{V}$-algebra $A$ and each increasing function $f: I \rightarrow A$, there is a homomorphism $h: L_{\mathcal{V}}(I) \rightarrow A$ such that $h \circ g=f$.

\section{Construction}

Now, we will determine the structure of $L_{\mathcal{V}}(I)$ in the particular case that the variety $\mathcal{V}$ is generated by $n$ algebras $S_{i}, n \in \mathbb{N}$, such that $C=\prod_{i=1}^{n} S_{i}$ is not an antichain.

Let $E$ be the set of all increasing functions from $I$ to the $\mathcal{V}$-algebra $C$ and let $g: I \rightarrow C^{E}$ be defined by $g(i)=G_{i}$, where $G_{i}(f)=f(i)$ for all $f \in E$ and $i \in I$. Then, $L=[G] \mathcal{V}$ is the free $\mathcal{V}$-algebra over $I$, where $G=\left\{G_{i}: i \in I\right\}$. Indeed, it follows easily that $i \leq j$ implies $G_{i} \leq G_{j}$ for all $i, j \in I$. On the other hand, suppose that there are $i, j \in I$ such that $G_{i} \leq G_{j}$ and $i \not \leq j$. Now, let us consider $a, b \in C, a<b$ and define $f^{*}: I \rightarrow C$ by

$$
f^{*}(k)= \begin{cases}b & \text { if } k \geq i \\ a & \text { otherwise. }\end{cases}
$$

Hence, we have that $f^{*} \in E, f^{*}(i)=b$ and $f^{*}(j)=a$. These statements imply that $G_{i}\left(f^{*}\right) \not \leq G_{j}\left(f^{*}\right)$, which is a contradiction. Thus, $g$ is an orderembedding. Besides, by the definition of $g$ we get that $G=g(I)$ and so, $L=[g(I)] v$. Therefore, (L1) holds.

Now we assume that $A$ is a $\mathcal{V}$-algebra and $f: I \rightarrow A$ is an increasing function. Since $\mathcal{V}$ is the variety generated by $C$, we have that $A$ is isomorphic to a subalgebra $A^{*}$ of $C^{X}$, where $X$ is an arbitrary set. Then, there is an isomorphism $\varphi: A \rightarrow A^{*}$ defined by the prescription $\varphi(a)=H_{a}$, where $H_{a} \in$ $C^{X}$ for all $a \in A$ and so, let us consider the function $\varphi^{*}=\varphi \circ f$, where $\varphi^{*}(i)=\varphi(f(i))=H_{f(i)}$. We claim that there is a homomorphism $h: L \rightarrow A^{*}$ such that $h \circ g=\varphi^{*}$. Indeed, for each $x_{0} \in X$ we define $\alpha_{x_{0}}: I \rightarrow C$ by $\alpha_{x_{0}}(i)=H_{f(i)}\left(x_{0}\right)$. Then, we infer that $\alpha_{x_{0}} \in E$. This assertion allows us to consider the function $k: X \rightarrow E$, defined by $k(x)=\alpha_{x}$ for all $x \in X$. Hence, it is routine to check that $h: L \rightarrow C^{X}$ where $h(F)=\bar{F}$ being $\bar{F}(x)=F(k(x))$ is a homomorphism. Moreover, we have that $(h \circ g)(i)=h\left(G_{i}\right)=\overline{G_{i}}$. Thus, for all $x \in X$ we infer that $\overline{G_{i}}(x)=G_{i}(k(x))=G_{i}\left(\alpha_{x}\right)=\alpha_{x}(i)=H_{f(i)}(x)=$ $\varphi^{*}(i)(x)$, which enable us to conclude that $(h \circ g)(i)=\varphi^{*}(i)$ for all $i \in I$. Finally, we have that $h(L) \subseteq A^{*}$. Indeed, since $L^{\prime}=\left\{F \in C^{E}: h(F) \in A^{*}\right\}$ is a $\mathcal{V}$-subalgebra of $C^{E}$ and $\bar{G}_{i} \in L^{\prime}$ for all $i \in I$, then $L \subseteq L^{\prime}$ and consequently $h(L) \subseteq A^{*}$. Therefore, (L2) holds. 
Remark 2. (i) If $I$ is an antichain, the notion introduced in Definition 1 coincides with the usual one of free algebra given in universal algebra.

(ii) From (i) and taking into account that we are dealing with varieties, Birkhoff's theorem (see [2]) should be verified. This means that $L_{\mathcal{V}}(I)$ $\in \mathcal{V}$, but this is not true. Indeed, let us consider the variety $\mathcal{V}(\mathbb{Z})$ generated by the set $\mathbb{Z}$ of all integers and let $J$ be the chain with two elements. Then, we have that $L_{\mathcal{V}}(J) \notin \mathcal{V}(\mathbb{Z})$.

(iii) If the algebra $C$, described as above, is an antichain then $L_{\mathcal{V}}(I) \in \mathcal{V}$ if and only if $I$ is an antichain.

In what follows we will apply the results obtained above to the varieties of 2 -valued implicative semilattices and that of Hilbert algebras generated by finite chains.

\section{2-valued implicative semilattices}

Notice that $(n+1)$-valued modal implicative semilattices were introduced and studied in [3]. Based on this notion, in $n=2$ case, we will call $S I_{2}$ algebras the one defined as algebras $\langle A, \rightarrow, \wedge, 1\rangle$ of type $(2,2,0)$ which verify the following identities:

$\left(\mathrm{IS}_{2} 1\right) x \rightarrow x=1$

$\left(\mathrm{IS}_{2} 2\right)(x \rightarrow y) \rightarrow y=y$,

$\left(\mathrm{IS}_{2} 3\right) x \rightarrow(y \wedge z)=(x \rightarrow z) \wedge(x \rightarrow y)$

$\left(\mathrm{IS}_{2} 4\right) x \wedge(x \rightarrow y)=x \wedge y$

$\left(\mathrm{IS}_{2} 5\right) \quad((x \rightarrow y) \rightarrow x) \rightarrow x=1$.

The variety of these algebras will be denoted by $\boldsymbol{I} \boldsymbol{S}_{\mathbf{2}}$ and it is generated by the algebra $\mathcal{S}=\langle\{0,1\}, \rightarrow, \wedge, 1\rangle$, where $0<1, x \wedge y$ is the infimum of $x, y$ and $\rightarrow$ satisfies the identities $0 \rightarrow x=1$ and $1 \rightarrow x=x$.

Our next aim is to describe the free $\boldsymbol{I} \boldsymbol{S}_{\mathbf{2}}$-algebra over a poset. From now on, if $X$ is poset and $Y \subseteq X$, we will denote by $[Y)$ the set of all $x \in X$ such that $y \leq x$ for some $y \in Y$.

Lemma 3. Let $L_{\boldsymbol{I} \boldsymbol{S}_{\mathbf{2}}}(I)$ be the free algebra over a poset $I$. Then, $L_{\boldsymbol{I} \boldsymbol{S}_{\mathbf{2}}}(I)=$ $\left[\bigwedge_{i \in I} G_{i}\right)$.

Proof. Let $F, H \in\left[\bigwedge_{i \in I} G_{i}\right)$. Then, taking into account that $H \leq F \rightarrow H$ we have that $F \rightarrow H \in\left[\bigwedge_{i \in I} G_{i}\right)$. Besides, $F \wedge H \in\left[\bigwedge_{i \in I} G_{i}\right)$ and so, $\left[\bigwedge_{i \in I} G_{i}\right) \in$ $\boldsymbol{I} \boldsymbol{S}_{\mathbf{2}}$. On the other hand, as $G_{i} \in\left[\bigwedge_{i \in I} G_{i}\right)$ for all $i \in I$ we conclude that $L_{\boldsymbol{I} \boldsymbol{S}_{\mathbf{2}}}(I) \subseteq\left[\bigwedge_{i \in I} G_{i}\right)$. The proof of the other inclusion is straightforward.

Let us consider the poset $I$ illustrated in Figure 1. 


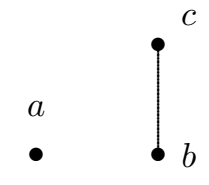

Figure 1

Then, the algebra $L_{\boldsymbol{I} \boldsymbol{S}_{\mathbf{2}}}(I)$ is the one indicated in Figure 2:

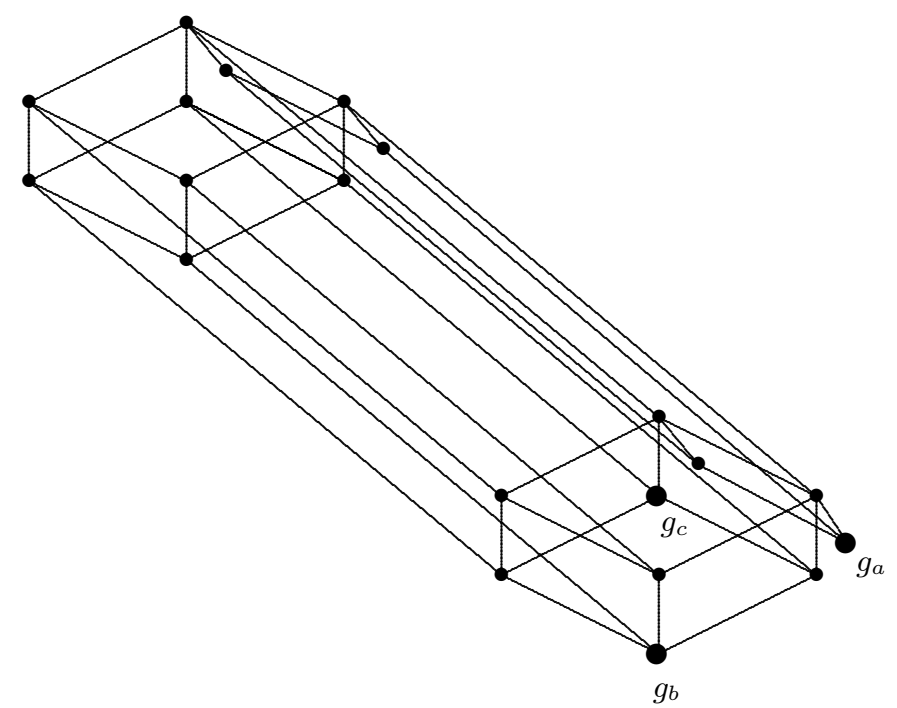

Figure 2

where $g_{a}, g_{b}$ and $g_{c}$ are the generators of this algebra.

Our next task is to determine the cardinal number of the free $I S_{2}$-algebra over a poset $I$ when $I$ is an antichain with $n$ elements and it will be denoted by $L_{\boldsymbol{I} \boldsymbol{S}_{\mathbf{2}}}(n)$.

From Lemma $3, L_{\boldsymbol{I} \boldsymbol{S}_{\mathbf{2}}}(n)$ is a Boolean algebra. Then, we have that

$$
L_{I S_{\mathbf{2}}}(n) \simeq \prod_{D \in \mathcal{\varepsilon}\left(L_{\boldsymbol{I} S_{\mathbf{2}}}(n)\right)} L_{\boldsymbol{I} \boldsymbol{S}_{\mathbf{2}}}(n) / D
$$

where $\mathcal{E}\left(L_{\boldsymbol{I} \boldsymbol{S}_{\mathbf{2}}}(n)\right)$ is the set of all maximal deductive systems of $L_{\boldsymbol{I} \boldsymbol{S}_{\mathbf{2}}}(n)$. Therefore,

$$
L_{I S_{2}}(n) \simeq B_{2}{ }^{\alpha},
$$

where $\alpha=\left|\mathcal{E}\left(L_{\boldsymbol{I} \boldsymbol{S}_{\mathbf{2}}}(n)\right)\right|$ is the cardinal number of $\mathcal{E}\left(L_{\boldsymbol{I} \boldsymbol{S}_{\mathbf{2}}}(n)\right)$ and $\boldsymbol{B}_{\mathbf{2}}$ is the Boolean algebra with two elements. Thus, in order to obtain $L_{\boldsymbol{I} \boldsymbol{S}_{\mathbf{2}}}(n)$ we will calculate $\alpha$. First, let us observe that $\bigwedge_{i \in I} G_{i} \notin M$ for all $M \in \mathcal{E}\left(L_{\boldsymbol{I} \boldsymbol{S}_{\mathbf{2}}}(n)\right)$. 
On the other hand, let $\beta: \operatorname{Epi}\left(L_{\boldsymbol{I} \boldsymbol{S}_{\mathbf{2}}}(n), \boldsymbol{B}_{\mathbf{2}}\right) \longrightarrow \mathcal{E}\left(L_{\boldsymbol{I} \boldsymbol{S}_{\mathbf{2}}}(n)\right)$ be the function defined by $\beta(H)=\operatorname{Ker}(H)$, where $\operatorname{Ker}(H)=\left\{f \in L_{\boldsymbol{I} \boldsymbol{S}_{\mathbf{2}}}(n): H(f)=1\right\}$. Then, it is simple to verify that $\beta$ is bijective and so, we infer that

$$
\left|\mathcal{E}\left(L_{\boldsymbol{I} \boldsymbol{S}_{\mathbf{2}}}(n)\right)\right|=\left|\operatorname{Epi}\left(L_{\boldsymbol{I} \boldsymbol{S}_{\mathbf{2}}}(n), \boldsymbol{B}_{\mathbf{2}}\right)\right| .
$$

Let $H \in \operatorname{Epi}\left(L_{\boldsymbol{I} \boldsymbol{S}_{\mathbf{2}}}(n), \boldsymbol{B}_{\mathbf{2}}\right)$ and $\bar{H}=\left.H\right|_{G}: G \rightarrow \boldsymbol{B}_{\mathbf{2}}$. Since $\bigwedge_{i \in I} G_{i} \notin$ $\operatorname{Ker}(H)$, we have that

$$
0=H\left(\bigwedge_{i \in I} G_{i}\right)=\bigwedge_{i \in I} \bar{H}\left(G_{i}\right) .
$$

Therefore, there is $i_{0}, 1 \leq i_{0} \leq n$, such that $\bar{H}\left(G_{i_{0}}\right)=0$. Then, if we consider the set

$$
F=\left\{f: G \rightarrow \boldsymbol{B}_{\mathbf{2}}: f\left(G_{i}\right)=0 \text { for some } i, 1 \leq i \leq n\right\}
$$

it follows that $\left|\operatorname{Epi}\left(L_{\boldsymbol{I} \boldsymbol{S}_{\mathbf{2}}}(n), \boldsymbol{B}_{\mathbf{2}}\right)\right|=|F|$ and so, we deduce that

$$
\alpha=|F|=2^{n}-1 .
$$

Therefore,

$$
L_{\boldsymbol{I} \boldsymbol{S}_{\mathbf{2}}}(n) \simeq \boldsymbol{B}_{\mathbf{2}}^{2^{n}-1} .
$$

Theorem 4. Let $L_{I S_{2}}(n)$ be the free $I S_{2}$-algebra over a poset I where $I$ is an antichain and $|I|=n$. Then $\left|L_{\boldsymbol{I} \boldsymbol{S}_{\mathbf{2}}}(n)\right|=2^{2^{n}-1}$.

\section{Hilbert algebras generated by finite chains}

A. Diego was one of the authors who made a fundamental contribution to the development of the theory of Hilbert algebras and his researches can be found in ([5]) (see also [4], [6]). From the results obtained by this author on the topic under consideration, it is worth mentioning the determination of the free Hilbert algebras with a finite set of free generators. Following A. Diego, by a Hilbert algebra we mean an algebra $\langle A, \rightarrow, 1\rangle$ of type $(2,0)$ satisfying the identities:

(H1) $x \rightarrow x=1$,

(H2) $1 \rightarrow x=x$,

(H3) $x \rightarrow(y \rightarrow z)=(x \rightarrow y) \rightarrow(x \rightarrow z)$,

(H4) $(x \rightarrow y) \rightarrow((y \rightarrow x) \rightarrow x)=(y \rightarrow x) \rightarrow((x \rightarrow y) \rightarrow y)$.

It is well known that by adding Ivo Thomas's identity:

(Tn) $T_{n}\left(x_{0}, \ldots, x_{n-1}\right)=\beta_{n-2} \rightarrow\left(\beta_{n-3} \rightarrow\left(\cdots \rightarrow\left(\beta_{0} \rightarrow x_{0}\right) \cdots\right)\right)=1$, where $\beta_{i}=\left(x_{i} \rightarrow x_{i+1}\right) \rightarrow x_{0}$ for all $i, 1 \leq i \leq n-2$,

to these algebras we obtain the variety of Hilbert algebras generated by a chain with $n$ elements which we will denote by $\mathcal{H}_{n}$. These algebras were considered by J. Berman and W. Blok in [1], where they showed the cardinal number of the finite free algebra with a finite set of free generators.

Now, taking into account the construction above indicated, we determine the algebra $L_{\mathcal{H}_{3}}(I)$. Figure 3 illustrated the poset $I$ and the algebra $C_{3}=\left\{0, \frac{1}{2}, 1\right\}$ which generates this variety: 


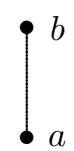

I

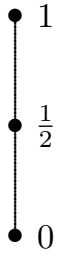

$C_{3}$

Figure 3

Then, the free algebra $L_{\mathcal{H}_{3}}(I)$ over the poset $I$ is the one indicated in Figure 4

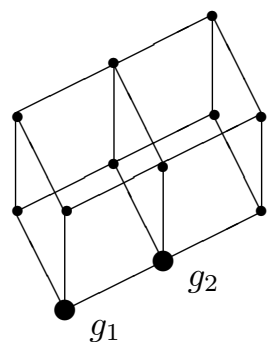

Figure 4

where $\mathrm{g}_{1}$ and $g_{2}$ are the free generators of this algebra.

Acknowledgements. A previous version of this paper appeared as a preprint at CLE e-Prints [8]. It was part of the M. Sc. Thesis [9] of the first author under the supervision of Prof. Alicia Ziliani.

\section{References}

[1] J. Berman and W. J. Blok, Free Lukasiewicz and hoop residuation algebras, Studia Logica 77 (2004), no. 2, 153-180.

[2] S. Burris and H. P. Sankappanavar, A Course in Universal Algebra, Graduate Texts in Mathematics, 78. Springer-Verlag, New York-Berlin, 1981.

[3] M. Canals Frau and A. V. Figallo, $(n+1)$-valued modal implicative semilattices, Proceedings of the 22nd International Symposium on Multipe Valued Logic. IEEE Computer Society (1992), 198-205.

[4] A. Diego, On implicative algebras, Rev. Un. Mat. Argentina 20 (1962), 310-311.

[5] _ Sobre las álgebras de Hilbert, Notas de Lógica Matemática 12, Univ. Nac. del Sur, Bahía Blanca, 1965.

[6] _ Sur les algébres de Hilbert, Collection de Logique Mathématique, Sér. A, Fasc. XXI Gauthier-Villars, Paris; E. Nauwelaerts, Louvain, 1966.

[7] R. P. Dilworth, Lattices with unique complements, Trans. Amer. Math. Soc. 57 (1945), $123-154$.

[8] A. Figallo, Jr., Free algebras over a poset in varieties generated by a finite number of algebras, CLE e-Prints of the Centre for Logic Vol. 9(3), 2009.

[9] _ Sobre la estructura ordenada de las álgebras de Hilbert, M.Sc. Thesis, Departamento de Matemática, Univerisdad Nacional del Sur, Argentina, 2006. 
Aldo Figallo, Jr.

Departamento de Matemática

Universidad Nacional del Sur

Bahía BlanCa 8000, Argentina

AND

Instituto de Ciencias BÁsicas

Universidad Nacional de San JuAN

Bahía Blanca 8000, Argentina

E-mail address: aldo.figallo@uns.edu.ar

Alicia Ziliani

Departamento de Matemática

Universidad Nacional del Sur

Argentina

AND

Instituto DE Ciencias BÁSICAS

Universidad Nacional de San Juan

Bahía Blanca 8000, Argentina

E-mail address: aziliani@criba.edu.ar 